\title{
Analysis of Medium Wrap Freehand Virtual Object Grasping in Exocentric Mixed Reality
}

\author{
Maadh AI-Kalbani, Ian Williams, and Maite Frutos-Pascual \\ DMT Lab, Birmingham City University
}
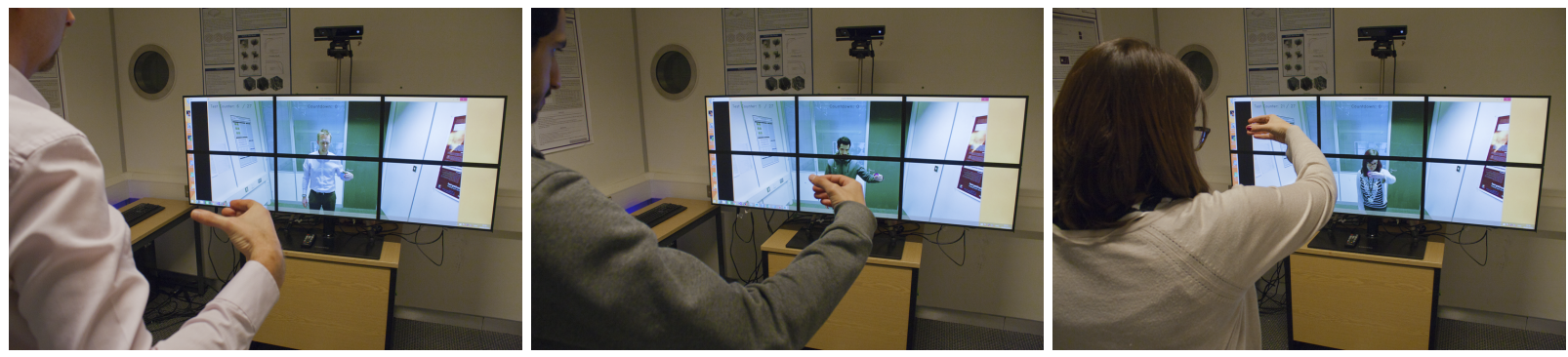

Figure 1: Participants performing medium wrap freehand grasping in an exocentric Mixed Reality environment

\begin{abstract}
This article presents an analysis into the accuracy and problems of freehand grasping in exocentric Mixed Reality (MR). We report on two experiments (1710 grasps) which quantify the influence different virtual object shape, size and position has on the most common physical grasp, a medium wrap. We propose two methods for grasp measurement, namely, the Grasp Aperture (GAp) and Grasp Displacement (GDisp). Controlled laboratory conditions are used where 30 right-handed participants attempt to recreate a medium wrap grasp. We present a comprehensive statistical analysis of the results giving pairwise comparisons of all conditions under test. The results illustrate that user Grasp Aperture varies less than expected in comparison to the variation of virtual object size, with common aperture sizes found. Regarding the position of the virtual object, depth estimation is often mismatched due to under judgement of the $\mathrm{z}$ position and $\mathrm{x}, \mathrm{y}$ displacement has common patterns. Results from this work can be applied to aid in the development of freehand grasping and considered as the first study into accuracy of freehand grasping in MR, provide a starting point for future interaction design.
\end{abstract}

Keywords: Grasping, Freehand Interaction, Natural Hand Interaction, Human Performance Measurement, Mixed Reality

Index Terms: H.5.1 [Information Interfaces and Presentation (e.g., HCI)]: Multimedia Information Systems-Artificial, augmented, and virtual realities; H.5.2 [Information Interfaces and Presentation (e.g., HCI)]: User Interfaces-; I.3.7 [Computer Graphics]: Three Dimensional Graphics and Realism-

\section{INTRODUCTION}

Grasping is one of the primary forms of manual interaction between humans and the physical world. While this is the case, the dexterous versatility of the human grasp poses many challenges for virtual object interaction and as such the objective quantification of these problems is largely unexplored.
Freehand grasping is defined as the physical manual grip between a human hand and a real object without utilisation of any wearable devices. Within Mixed Reality (MR), freehand grasping is the manual grip between a (real) user and a (virtual) object without the utilisation of wearable sensors. In many applications this form of interaction is preferable due to the discomfort of wearable devices (Suzuki et al. [1]) and the often time consuming configuration and user adaptation (Holz et al. [2]) of them. Moreover, recent studies notably Ponto et al. [3], have illustrated that wearable methods of user feedback, notably biofeedback or electromyograms (EMG), can aid in human grasping, but often cause fatigue and discomfort.

Early developments of freehand interaction largely ignored the application of complex human grasps. The work of Rekimoto, Wu and Balakrishnan [4, 5], presented four interaction techniques, including grasping, applied within a projected tabletop MR environment. Within this work, the authors consider quantifiable measurements of hand accuracy and error distance (hand to surface), however no analysis of human grasping accuracy was presented. More recently, freehand interactions were presented by Benko et al. [6] covering holding, moving and knocking down motions. Usability evaluation is presented, although no consideration is given to the fundamental human physical grasp parameters.

Within this work we present an analysis of freehand grasping with virtual objects. We illustrate the common errors when users grasp virtual objects in an exocentric MR scene. We show the common patterns to human grasping as quantifiable measures of both Grasp Aperture and Grasp Displacement. Consideration is given to the size, shape and position of the virtual objects and we employ a rigorous experiment methodology to quantify the user's grasp differences. Finally we present the measures useful for application within interactive grasping systems, thus enabling translation of these results to improved interaction design. The rest of this paper is structured as follows: Section 2 details the theories of freehand grasping and the current methods for objective measurements of grasping interaction. Section 3 then presents the models for grasping, showing measures for Grasp Aperture and Grasp Displacement. Section 4 presents the two experiment designs for our study, detailing the participants used, the protocol employed, the system utilised and the statistical analysis. Section 5 then presents the results from Experiment 1 , quantifying the difference object size and shape has on freehand grasp accuracy, while section 6 presents Experiment 2 quantifying the impact of $\mathrm{x}, \mathrm{y}$ and $\mathrm{z}$ position of a virtual object. Section 7 concludes the work presenting routes for further analysis. 


\section{Related Work}

\subsection{Grasping}

Feix et al. [7] define a grasp as being every static posture at which an object can be held securely with a single hand. Furthermore MacKenzie and Iberall [8] summarise phases of a grasp strategy by: gathering perceptual information about an object's intrinsic and extrinsic properties, followed by configuration of the hand during motion for placement on an object and finalised by maintaining and establishing a stable grasp once contact with the object is made.

Derived from the complexity and physiology of the human hand, the grasping process requires various simplifications to make it easier to understand (Cutkosky and Howe [9]). Human grasp taxonomies presented in $[10,11,12,13]$ aim to simplify the grasp complexity into 33 classified grasp types. More recent work has developed this classification further, notably Bullock et al. [14] and Feix et al. [15] who classify the most used human grasps on real physical objects into five versatile grasps that can be used on most physically graspable objects, with the most common being the medium wrap.

\subsection{Grasping Virtual Objects}

Mixed or Augmented Reality (MR/AR) applications, where virtual objects overlay a real scene, present prominent research into freehand interactions. Early work $[16,17]$ in AR technology addressed occlusion problems in two dimensional AR interactions using freehand interaction techniques such as dragging and dropping. Gestural interactions with virtual objects, which included grasping, were also developed by tracking the index and thumb fingers, however grasp accuracy was not addressed. Moreover, both studies indicated that users found these systems intuitive and easy to use without the use of a formal evaluation study. More recently, Bai et al. [18] developed gestures that emulate human grasping, computed in a $3 \mathrm{D}$ handheld AR interface. Even though a formal usability study was employed to assess performance, time was the the only metric used. Datcu and Lukosch [19] also presented AR freehand interactions in a crime scene investigation application, showing novel methods in freehand interactions, however the analysis of accuracy was limited to pointing interactions only. Freehand grasping in an AR context, without the use of wearable devices, has been presented in [1]. Findings from this work stated that the freehand interaction alongside the visual feedback increased the feel of grasping for users. However, no results or comprehensive analysis was provided to support this claim. More recently Cidota et al. [20] assessed upper extremity motor dysfunction using freehand interactions in the context of a serious AR game, the study was aimed at subjectively evaluating the usability of the system developed for users rather than the objective measurements of grasp accuracy.

\subsection{Measuring Interaction}

Many performance metrics exist for evaluating usability in MR, yet measuring the accuracy of grasping and understanding the perceptual nature of freehand grasping of virtual objects has not yet been explored. Swan et al. [21] conducted a survey of user-based experimentation in $\mathrm{AR}$, measuring interaction methods, which was divided into areas of perception, performance and collaboration.

Usability evaluation metrics are also frequently used in MR [22], to quantify how easily and consistently users are able to perform tasks. Furthermore a perceptual study by Swan et al. [23] measured depth judgements in matching and reaching interactions by calculating user distance from an ideal target location. User hand distance within interaction was also explored by Hough et al. [24] who quantified the fidelity and plausibility of bi-manual interactions in a virtual studio MR environment. Fidelity of interaction in standard and immersive displays was evaluated using subjective measurements to quantify feel of presence and engagement McMahan et al. [25]. A rehabilitation system using virtual reality aimed at improving hand function of stroke patients that was developed by Jack et al. [26] used success rate across various tasks to test different hand functions. Speed, fraction and strength are used as a measure of interaction.

Even though user evaluation metrics only form a small margin of evaluation methods in MR [22], they have been growing in the past few years. However, designing new metrics that quantify the accuracy and nature of freehand grasping of virtual objects is still required for our work, as evaluation of this kind of interaction in a MR context has not yet been explored.

\section{GRASP MODEL}

In this work we focus on the last phase of a grasp defined in the work of [8], namely that users "maintain and establish a stable grasp". For this we assess one grasp type, the medium wrap, defined in $[14,15]$ as the most common manual human grip (see Fig. 2a).

\subsection{Grasp Aperture}

To measure how accurately users estimate the size of the virtual object the aperture of a users grasp is applied, based on the work of Edsinger and Kemp [27]. Here we define the Grasp Aperture $(G A p)$ in Equation 1 to be the distance between a users thumb tip and index finger tip (see Fig. 2b).

$$
G A p=\sqrt{\left(P_{x}-B_{x}\right)^{2}+\left(P_{y}-B_{y}\right)^{2}+\left(P_{z}-B_{z}\right)^{2}}
$$

Where $P_{x}, P_{y}$ and $P_{z}$ are the co-ordinates of the index finger tip, and $B_{x}, B_{y}$ and $B_{z}$ are co-ordinates of the thumb tip.

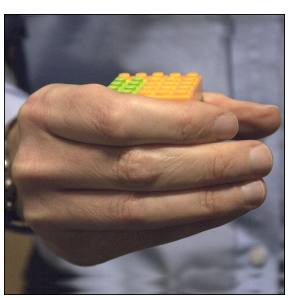

(a)

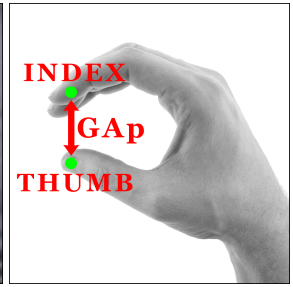

(b)

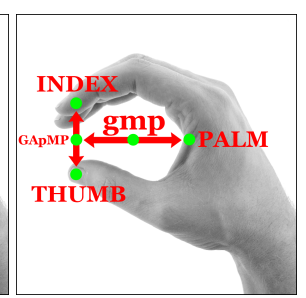

(c)
Figure 2: Grasp Measurements: (a) medium wrap grasp, (b) Grasp Aperture $(G A p)$ and (c) grasp middle point (gmp)

\subsection{Grasp Displacement}

As a grasp requires users to estimate both the size of the virtual object and the spatial position, the Grasp Aperture ( $G A p$ ) would not be a suitable measure if used alone. Therefore to measure the position accuracy of the user's hands against the virtual object we define a measure of the grasp middle point $(g m p)$. Here we define $g m p$ in Equation 2 as the position in the grasp relating middle point between the Grasp Aperture Middle Point $(G A p M P)$ and the users palm (see Fig. 2c).

$$
g m p=\left(\frac{G A p M P_{x}+p a l m_{x}}{2}, \frac{G A p M P_{y}+p a l m_{y}}{2}, \frac{G A p M P_{z}+p a l m_{z}}{2}\right)
$$

Where $\operatorname{palm}_{x}$, palm $_{y}$ and palm $z$ are the co-ordinates of the users palm and GApMP is the middle point in the grasp. Grasp Displacement (GDisp) is defined as the difference between the co-ordinates of the virtual object middle point ( $\mathrm{omp}$ ) from the gmp. 


\section{EXPERIMENT DESIGN}

Two experiments are reported in this study. Experiment 1 to quantify the influence of object size and object type (cube and sphere), and Experiment 2 to test the influence of object position in $\mathrm{x}, \mathrm{y}$ and $\mathrm{z}$ space on grasp accuracy. Conditions of both experiments are shown in Table 1, with the accuracy of a medium wrap grasp measured against Grasp Aperture (GAp) and Grasp Displacement (GDisp) (see section 3). To represent the accuracy of a grasp independent of additional rendering, for both experiments, we use objects which have not undergone complex rendering and represent a simple abstract shape.

Table 1: Experiments 1 and 2 conditions, where $\mathrm{x}$ is measured from the midpoint of the sensor, $\mathrm{y}$ from ground and $\mathrm{z}$ from sensor. Here we define Left, Middle and Right to be horizontal positions, and Top, Centre and Bottom to be vertical positions

\begin{tabular}{|c|c|c|c|c|}
\hline \multicolumn{5}{|c|}{ Experiment 1} \\
\hline Condition & \multicolumn{4}{|c|}{ Levels } \\
\hline Size & \multicolumn{4}{|c|}{$40 \mathrm{~mm}, 50 \mathrm{~mm}, 60 \mathrm{~mm}, 70 \mathrm{~mm}, 80 \mathrm{~mm}, 100 \mathrm{~mm}$} \\
\hline Position (z) & \multicolumn{4}{|c|}{$(1600 \mathrm{~mm})$ as shown in Fig. 3a } \\
\hline Object Type & \multicolumn{4}{|c|}{ Cube and Sphere } \\
\hline \multicolumn{5}{|c|}{ Experiment 2} \\
\hline Condition & \multicolumn{4}{|c|}{ Levels } \\
\hline \multirow[t]{4}{*}{ Position $(\mathrm{x}, \mathrm{y})$} & & LEFT & MIDDLE & RIGHT \\
\hline & TOP & $(-40,1290)$ & $(0,1290)$ & $(40,1290)$ \\
\hline & CENTRE & $(-40,1250)$ & $(0,1250)$ & $(40,1250)$ \\
\hline & BOTTOM & $(-40,1210)$ & $(0,1210)$ & $(40,1210)$ \\
\hline Position $(\mathrm{z})$ & \multicolumn{4}{|c|}{$(1400 \mathrm{~mm}),(1600 \mathrm{~mm})$ and $(1800 \mathrm{~mm})$ as shown in Fig. $3 \mathrm{l}$} \\
\hline Object Type & \multicolumn{4}{|c|}{ Cube and Sphere } \\
\hline
\end{tabular}

\subsection{Participants}

A total of 30 participants from a population of university students and staff members volunteered to take part in this study. Participants completed a standardized consent form, were not compensated and all data collected was anonymised. Visual acuity was measured using a Snellen chart and each participant was required to pass an Ishihara test to exclude for colour blindness. No participants suffering from colour blindness and/or with visual acuity of $<0.80$ were included in the analysis. Height, arm length and hand size of all participants were measured prior to each experiment, to ensure that aspects of the experimental design are within the biomechanical reach of participants. All participants were right handed [28].

Participants ranged in age from 19 to $62(M=30.43, \mathrm{SD}=9.78)$, in arm length from $480 \mathrm{~mm}$ to $660 \mathrm{~mm}(M=552.40, \mathrm{SD}=43.80)$, in hand size from $160 \mathrm{~mm}$ to $200 \mathrm{~mm}(M=186.80, \mathrm{SD}=10.40)$, in height from $1570 \mathrm{~mm}$ to $1950 \mathrm{~mm}(M=1744.00, \mathrm{SD}=90.00)$ and 6 were female and 24 male. Taking into account balance in hand size, arm length, gender, age and height, we separated participants into two groups of 15 for each experiment.

\subsection{System Architecture}

The system developed integrated the use of a Microsoft Kinect 2, a (HD) video camera, and a SyncMasterX $6^{1}$ feedback monitor. The experiments were developed in $\mathrm{C}++$ using Kinect SDK. Autodesk Maya $^{2}$ was used for modelling 3D objects, open computer vision library OpenCV $\mathrm{V}^{3}$ for video processing and open graphics library

\footnotetext{
${ }^{1}$ http://www.samsung.com/us/support/owners/product/MD230X6

${ }^{2}$ http://www.autodesk.com/products/maya/overview

${ }^{3} \mathrm{http}: / /$ opencv.org/
}

OpenGL ${ }^{4}$ for real time reading, loading and texturing of three dimensional (3D) virtual objects.

The physical configuration of the system strictly followed the recommendations of Kinect 2 manufacturers ${ }^{5}$. Participants stood $2000 \mathrm{~mm}$ away from the sensor under controlled and constant lighting conditions, the sensor was placed at a height of $1800 \mathrm{~mm}$ and tilted at an angle of $13.78^{\circ}$ to show the working space around participants and to eliminate any significant self occlusion problems (see Fig. 3 and Fig. 1)

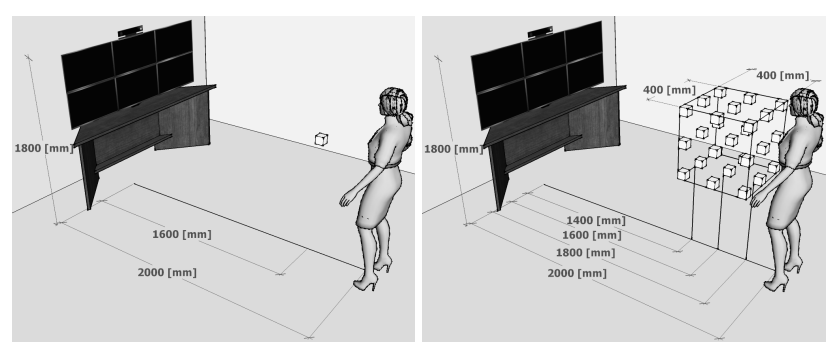

(a)

(b)

Figure 3: Freehand interaction setup: (a) Experiment 1, (b) Experiment 2. Conditions are given in Table 1

\subsection{Experimental Protocol}

Participants were naive to the purposes of the experiments, but their level of experience in MR systems ranged from novice to expert. Participants stood $2000 \mathrm{~mm}$ away from the monitor (size: $62 \mathrm{in}$ $\times 30$ in, resolution: $5760 \times 2160$ ), displaying a composited real time mirrored scene overlaying virtual objects with the video feed. Grasping parameters (GAp, GDisp) are measured from the sensor, not to test biomechanics of the hand but to quantify errors in spatial positioning and aperture estimation.

Participants underwent initial training of the medium wrap grasp on real and virtual objects. The test coordinator explained the procedure between each block of tests (i.e cube and sphere), and participants were allowed to rest before presentation of every object. Each experiment was formed of a 5 minutes training/instruction session, 10 minutes of grasping a cuboid object, 5 minutes break and 10 minutes of grasping a spherical object.

During the experiment all participants were instructed to verbally inform the test coordinator that they are satisfied with the grasp they have performed, and maintain the grasp for 5 seconds while the measurements for that condition are stored.

\subsection{Statistical Analysis}

Kruskal Wallis H test [29] is used for analysis over the ANOVA test as a Shapiro-Wilk test for normality showed that our data did not follow a normal distribution [30]. A post-hoc test for multiple comparisons using Dunn Test with Bonferroni correction [31] is preferred to a Mann-Whitney U post hoc test [32], and is used for statistically significant results of the Kruskal Wallis $\mathrm{H}$ test, to check for statistical differences using pairwise comparisons between groups of independent variables. Jonckheere-Terpstra [33, 34], a nonparametric test, is used when required to determine if there are any statistically significant trends between ordinal independent variables and continuous dependent variables [30].

\footnotetext{
${ }^{4}$ http://www.opengl.org/

${ }^{5}$ http://support.xbox.com/en-GB/xbox-360/kinect/kinect-sensor-setup
} 


\section{Experiment 1: OBJect Size ANd Shape}

We used a $2 \times 6$ within-subjects design, with two primary conditions: object size and object type (see Table 1). All 15 participants took part in both conditions. Every permutation for both object types was randomly presented to participants to exclude potential learning effects. In total, each participant completed 6 (sizes) $\times 5$ (repetitions) $\times 2$ (objects $)=60$ trials and 900 grasps $(60$ trials $\times 15$ participants). Each static grasp of every participant was recorded for 5 seconds (75 frames), leading to collecting 67500 raw data points (900 grasps $\times 75$ frames).

Hypothesis: We test the null hypotheses that changes in object size have no effect on a) Grasp Aperture and b) Grasp Displacement

\subsection{Procedure}

Participants were instructed to accurately match their medium wrap Grasp Aperture to the size and position of the virtual object in the shortest time possible. During the experiment, an object (cube or sphere) appeared on the feedback monitor, in 6 different sizes (see Table 1). Objects were positioned $1600 \mathrm{~mm}$ away from the sensor and $400 \mathrm{~mm}$ away from participants (z), at a height of $1250 \mathrm{~mm}(\mathrm{y})$ and at the zero (x) point on the sensor. This position was constant throughout the experiment (see Fig 3a).

\subsection{Results and Analysis}

Table 2: Significant Post-hoc Pairwise Comparisons: Experiment 1

\begin{tabular}{|c|c|c|c|c|c|c|}
\hline Sizes & 40 & 50 & 60 & 70 & 80 & 100 \\
\hline 40 & & - $\Delta \bullet$ & $\bullet \circ \Delta \triangle \bullet \quad$ । & $\bullet \circ \Delta \triangle \diamond \diamond$ & $\bullet \circ \Delta \Delta \diamond \diamond \quad \square$ & $\bullet \circ \Delta \Delta \diamond \diamond$ \\
\hline 50 & & & $\bullet \circ \quad \Delta \diamond \diamond$ & $\bullet \circ \Delta \Delta \diamond \diamond \square \square$ & $\bullet \circ \Delta \triangle \diamond \diamond$ & $\bullet \circ \Delta \Delta \diamond \diamond$ \\
\hline 60 & & & & $\bullet \circ \Delta \Delta \diamond \diamond \square$ & $\bullet \circ \Delta \triangle \diamond \diamond$ & $\bullet \circ \Delta \Delta \diamond \diamond$ \\
\hline 70 & & & & & $\Delta \quad$ I & $\bullet \circ \Delta \Delta \diamond \diamond \mathbf{\square}$ \\
\hline 80 & & & & 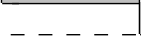 & & $\bullet \circ \quad \Delta \bullet \diamond$ \\
\hline 100 & & & & & & \\
\hline
\end{tabular}

Symbols represented in constant $\operatorname{order}(\bullet \Delta \Delta \diamond \diamond \square \square)$ show significance in a post-hoc Dunn Test with Bonferroni correction using an $\alpha$ level of 0.01 for the following: $-G A p$ Cube, $\circ G A p$ - Sphere, $\Delta$ GDisp $_{\mathrm{x}}$ - Cube, $\triangle$ GDisp $p_{\mathrm{x}}$ - Sphere, GDisp $p_{\mathrm{y}}$ - Cube, $\diamond G D i s p_{\mathrm{y}}-$ Sphere, GDisp $_{\mathrm{z}}$ - Cube, $\square$ GDisp - Sphere. No symbols indicate statistical similarity

\subsubsection{Results - Grasp Aperture (GAp)}

Statistically significant differences in Grasp Aperture $(G A p)$ between different object types in different sizes $\left(\chi^{2}(1)=2028, p<\right.$ 0.01 ) were found. Statistically significant differences in $G A p$ between different cube sizes $\left(\chi^{2}(5)=2824, p<0.01\right)$ and different sphere sizes $\left(\chi^{2}(5)=1477, p<0.01\right)$ were also found. Significant adjusted post-hoc results are reported in Table 2 (see $\bullet$ for cubes, O for spheres). Regardless of similarity of $G A p$ to object size, participants increased their $G A p$ as object size increased.

In order to understand the practical significance of the multiple comparisons in our post-hoc analysis, Cohen's $d$ [35] effect size for independent t-tests is calculated. For both objects, majority of effects in the multiple comparison were medium $(d<0.50)$ and small $(d<0.20)$

Lowest mean difference between $G A p$ and object size was in the $80 \mathrm{~mm}$ size for cubes $(M=80.13 \mathrm{~mm}, \mathrm{SD}=24.58)$, and in the $70 \mathrm{~mm}$ size for spheres $(M=72.18 \mathrm{~mm}, \mathrm{SD}=26.03)$. Highest mean difference between $G A p$ and object size was present in the $40 \mathrm{~mm}$ size for cubes $(M=66.31 \mathrm{~mm}, \mathrm{SD}=29.97)$, and spheres $(M=65.73 \mathrm{~mm}, \mathrm{SD}$ $=30.83$ ).

A linear relationship is present between $G A p$ and object size in the context of grasping real objects [8], thus the correlation between $G A p$ and object size using a Jonckheere-Terpstra test for ordered alternatives shows a statistically significant trend of higher $G A p$ with higher levels of cube size $\left(\mathrm{T}_{\mathrm{JT}}=2.88 \times 10^{8}, \mathrm{z}=53.58, p<0.01\right)$ and sphere size $\left(\mathrm{T}_{\mathrm{JT}}=2.75 \times 10^{8}, \mathrm{z}=36.65, p<0.01\right)$. This indicates that a positive trend between $G A p$ and cube sizes is present in freehand grasping of virtual objects.

\subsubsection{Findings - Grasp Aperture $(G A p)$}

For both objects, participants overestimated object size up until the size that had the lowest mean difference between $G A p$ and object size $(80 \mathrm{~mm}$ for the cube and $70 \mathrm{~mm}$ for the sphere). In addition, both objects showed that with the $100 \mathrm{~mm}$ size, participants underestimated its size by a mean of $-11.23 \mathrm{~mm}$ for cubes $(\mathrm{SD}=22.39$ ), and $-22.76 \mathrm{~mm}$ for spheres $(\mathrm{SD}=24.35)$.

Participants had lower mean $G A p$ in grasping spheres $(M=$ $69.25 \mathrm{~mm}, \mathrm{SD}=28.27)$ than cubes $(M=77.64 \mathrm{~mm}, \mathrm{SD}=26.36)$. Mean difference between $G A p$ and size was lower in 4 of the 6 sizes $(40 \mathrm{~mm}, 50 \mathrm{~mm}, 60 \mathrm{~mm}$ and $70 \mathrm{~mm})$ for spheres than cubes.

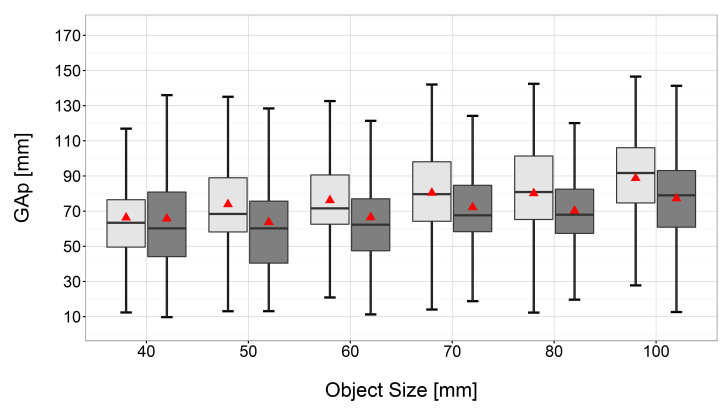

Figure 4: GAp for different object sizes in the $1600 \mathrm{~mm}$ z plane. Light grey boxplots show cubes, and dark grey boxplots show spheres. Red triangles on boxplots indicate the mean GAp across all participants for each size. Whiskers represent the highest and lowest values within 1.5 times the interquartile range from the lower and upper quartiles

Fig. 4 shows that the mean $G A p$ ranged from $65.70 \mathrm{~mm}(\mathrm{SD}=$ $30.83)$ to $88.80 \mathrm{~mm}(\mathrm{SD}=22.39)$ across all sizes of both objects. This range shows that $G A p$ in grasping virtual objects is between $65.70 \mathrm{~mm}$ to $88.80 \mathrm{~mm}$ regardless of object size or type. No direct linear relationship between object size and $G A p$ as in real objects was found.

Mean completion time ranged from 3.23 ( $\mathrm{SD}=1.29$ ) to $4.48 \mathrm{sec}-$ onds $(\mathrm{SD}=1.96)$ for both objects. Shortest completion time was present in the $80 \mathrm{~mm}$ size for both objects, this could be an indication that the $80 \mathrm{~mm}$ object size (with the lowest mean difference between $G A p$ and object size) felt the most natural graspable size for participants. Even though statistically significant differences in completion time between different cube sizes $\left(\chi^{2}(5)=449, p<\right.$ $0.01)$ and sphere sizes $\left(\chi^{2}(5)=572, p<0.01\right)$ were found, all effect sizes were small $(d<0.30)$ or negligible $(d<0.20)$, and no trends between completion time and object size were found.

As statistically significant results were found for both size and object types conditions, the null hypothesis that the conditions do not have an effect on $G A p$ is rejected. GAp is affected in our study by changes in size and object type.

\subsubsection{Results - Grasp Displacement (GDisp)}

Statistically significant differences in Grasp Displacement in the $\mathrm{x}$ axis $\left(G D i s p_{\mathrm{x}}\right)$ between different object types in different sizes $\left(\chi^{2}(1)=42730, p<0.01\right)$ were found. Statistically significant differences in GDisp $p_{\mathrm{x}}$ between different cube sizes $\left(\chi^{2}(5)=922, p<\right.$ $0.01)$ and different sphere sizes $\left(\chi^{2}(5)=2728, p<0.01\right)$ were also 

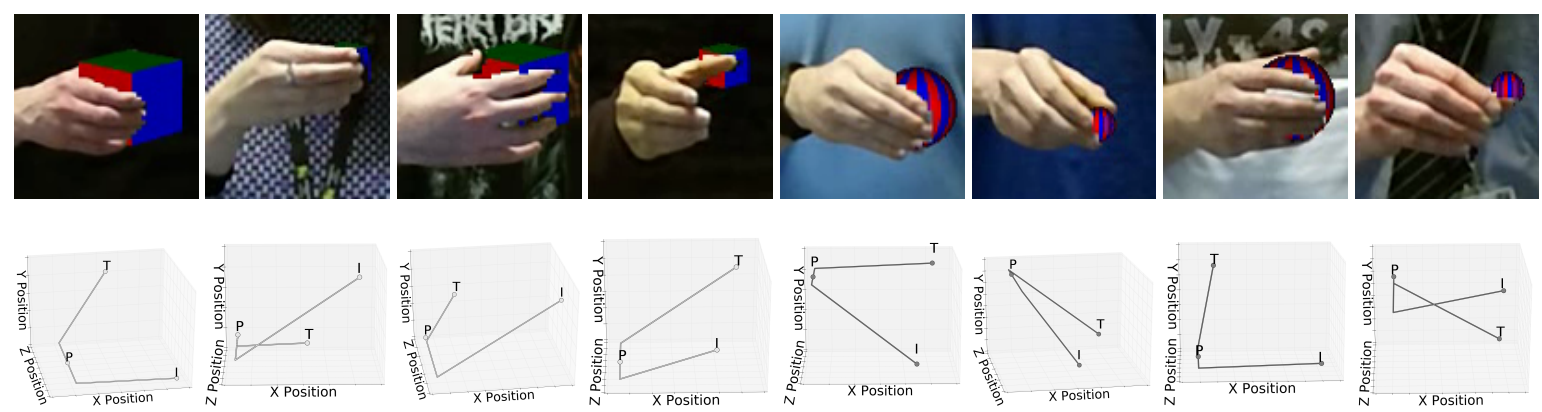

Figure 5: Top row: examples of grasp variation in Experiment 1 across participants in the 40mm and 100mm sizes of cubes and spheres. Bottom row: plots that visualise the grasps presented in the top row in 3D space, letters in plots indicate: T - Thumb finger, I - Index/Tip finger and P - Palm

found. Significant adjusted post-hoc results are reported in Table 2 (see $\Delta$ for cubes, $\Delta$ for spheres).

For both objects, majority of effects in the multiple comparison were medium $(d>0.50)$ and small $(d>0.20)$.

Participants had the highest mean GDisp $p_{\mathrm{x}}$ in the $40 \mathrm{~mm}$ size for cubes $(M=31.45 \mathrm{~mm}, \mathrm{SD}=14.10)$ and spheres $(M=36.51 \mathrm{~mm}$, $\mathrm{SD}=13.31)$. Lowest mean GDisp $p_{\mathrm{x}}$ was present in the $70 \mathrm{~mm}$ size for cubes $(M=25.34 \mathrm{~mm}, \mathrm{SD}=14.75)$, and in the $100 \mathrm{~mm}$ size for spheres $(M=24.56 \mathrm{~mm}, \mathrm{SD}=14.26)$.

Statistically significant differences in Grasp Displacement in the $\mathrm{y}$ axis $\left(G D i s p_{\mathrm{y}}\right)$ between different object types in different sizes $\left(\chi^{2}(1)=50448, p<0.01\right)$ were found. Statistically significant differences in GDisp $p_{\mathrm{y}}$ between different cube sizes $\left(\chi^{2}(5)=1556, p<\right.$ $0.01)$ and sphere sizes $\left(\chi^{2}(5)=1845, p<0.01\right)$ were also found. Significant adjusted post-hoc results are reported in Table 2 (see for cubes, $\diamond$ for spheres).

For both objects, majority of effects in the multiple comparison were medium $(d>0.50)$ and small $(d>0.20)$.

Participants had the highest mean GDisp $p_{\mathrm{y}}$ in the $40 \mathrm{~mm}$ size for cubes $(M=-15.81 \mathrm{~mm}, \mathrm{SD}=12.15)$, and in the $50 \mathrm{~mm}$ size $(M=$ $-12.82 \mathrm{~mm}, \mathrm{SD}=13.03$ ) for spheres. Lowest mean GDisp $p_{\mathrm{y}}$ was present in the $100 \mathrm{~mm}$ size for cubes $(M=-8.90 \mathrm{~mm}, \mathrm{SD}=12.36)$ and spheres $(M=-5.77 \mathrm{~mm}, \mathrm{SD}=10.39)$.

Statistically significant differences in Grasp Displacement in the $\mathrm{z}$ axis $\left(G D i s p_{\mathrm{z}}\right)$ between different object types in different $\operatorname{sizes}\left(\chi^{2}(1)=364, p<0.01\right)$ were found. Statistically significant differences in GDisp $p_{\mathrm{z}}$ between different cube sizes $\left(\chi^{2}(5)=135\right.$, $p<0.01)$ and sphere sizes $\left(\chi^{2}(5)=82.77, p<0.01\right)$ were also found. Significant adjusted post-hoc results are reported in Table 2 (see $\square$ for cubes, $\square$ for spheres).

Effect sizes for both objects were all negligible $(d<0.20)$. This was expected as the $\mathrm{z}$ position of the different cubes and spheres was unchanged throughout the experiment.

\subsubsection{Findings - Grasp Displacement (GDisp)}

Positive GDisp $p_{\mathrm{x}}$ was present for both objects. This positive $G D i s p_{\mathrm{x}}$ is expected, as all participants in this study were right handed, and the grasp middle point ( $\mathrm{gmp}$ ) is computed on the right side of the virtual object. Across all sizes and participants, lower GDis $p_{\mathrm{x}}$ in grasping cubes $(M=28.01 \mathrm{~mm}, \mathrm{SD}=14.08)$ than spheres $(M=31.52 \mathrm{~mm}, \mathrm{SD}=14.67)$ was found, meaning higher GDisp $p_{\mathrm{x}}$ was present for spheres.

Bounds of clusters presented in Fig. 6a and Fig. 6b show similarity of gmp placement for all cube and sphere sizes. Mean GDisp ranged from $25.35 \mathrm{~mm}(\mathrm{SD}=14.75)$ to $31.45 \mathrm{~mm}(\mathrm{SD}=14.10)$ across all cube sizes, and from $24.56 \mathrm{~mm}(\mathrm{SD}=14.26)$ to $36.51 \mathrm{~mm}$
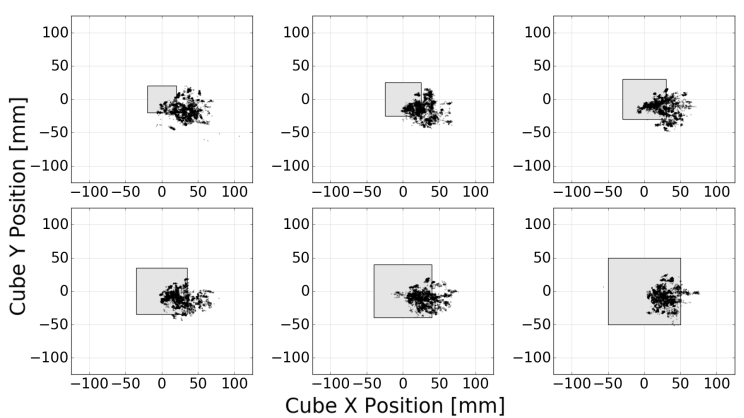

(a)
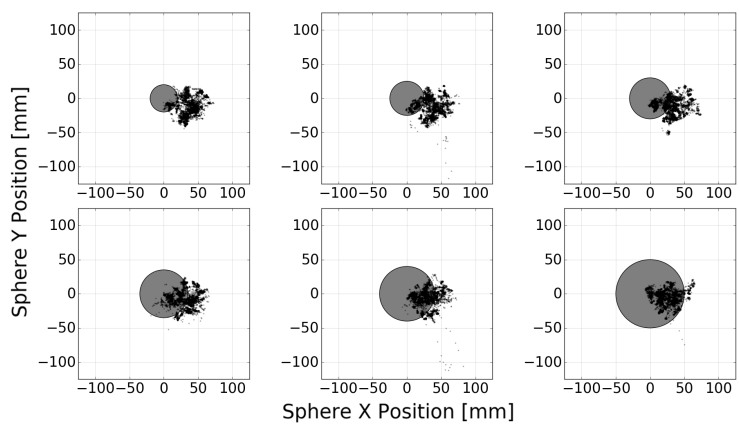

(b)

Figure 6: gmp placement (black clusters) in the $\mathrm{x}$ and $\mathrm{y}$ axes of all participants for Experiment 1: (a) cube, and (b) sphere where sizes from top left: $40 \mathrm{~mm}-50 \mathrm{~mm}-60 \mathrm{~mm}-70 \mathrm{~mm}-80 \mathrm{~mm}-100 \mathrm{~mm}$

$(\mathrm{SD}=13.31)$ across all sphere sizes, showing a higher SD for cubes. However, SD differences within object sizes between cubes and spheres were comparable, indicating that contact of the $g m p$ with the surface of the object was reflective of size growth of objects rather than movements by participants.

Negative GDisp $p_{\mathrm{y}}$ was present for both objects. This reveals that participants placed their gmp below the object middle point (omp) for both cube and sphere. Interestingly, participants chose a lower point to the omp and not a higher one, this is potentially attributed to participants trying to show parts of the objects presented to them on the feedback monitor. Clusters in Fig. 6a and Fig. 6b show that gmp placement was comparable for all cube and sphere sizes along the y axis. Mean GDisp y ranged from $-15.81 \mathrm{~mm}(\mathrm{SD}=12.15)$ to $-8.90 \mathrm{~mm}(\mathrm{SD}=12.36)$ in all cube sizes, and from $-12.82 \mathrm{~mm}$ (SD $=12.76)$ to $-5.77 \mathrm{~mm}(\mathrm{SD}=10.39)$ in all sphere sizes, showing a higher SD for spheres. Across all sizes, participants had lower mean 
GDisp $p_{\mathrm{y}}$ in grasping spheres $(M=-9.84 \mathrm{~mm}, \mathrm{SD}=12.51)$ than cubes $(M=-12.37 \mathrm{~mm}, \mathrm{SD}=11.94)$. However, SD differences within object sizes between cubes and spheres were comparable.

Out of all three axes, GDisp z $_{\mathrm{z}}$ presented the highest displacement. This was expected as only visual feedback was used in this exocentric setting through utilising a single monitor.
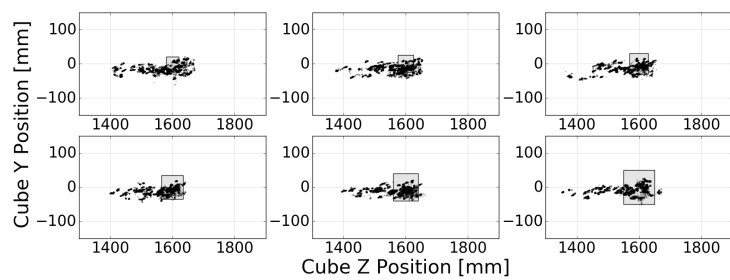

(a)
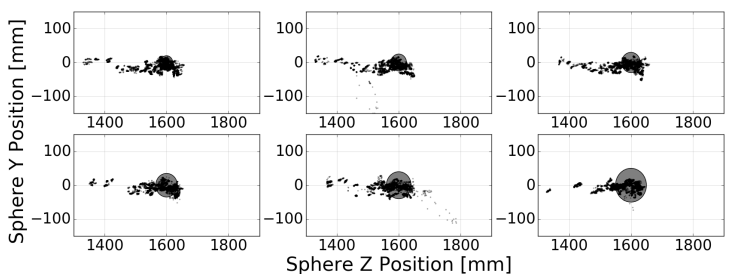

(b)

Figure 7: gmp placement (black clusters) in the $\mathrm{z}$ axis of all participants for Experiment 1: (a) cube, and (b) sphere where sizes from top left: $40 \mathrm{~mm}-50 \mathrm{~mm}-60 \mathrm{~mm}-70 \mathrm{~mm}-80 \mathrm{~mm}-100 \mathrm{~mm}$

Due to the feedback modality in our work, the terms underestimation and overestimation are opposite to those of depth perception, hence in this study, depth refers to the distance from the feedback monitor and not the user as in depth perception studies. Negative $G D i s p_{\mathrm{z}}$ was found in both objects across all sizes, this indicates that majority of participants underestimated the $\mathrm{z}$ position of the $\mathrm{omp}$ by placing their $g m p$ in front of the $o m p$ for all sizes, as shown in Fig. $7 \mathrm{a}$ and $7 \mathrm{~b}$. Overestimation was also present, but not as frequent as underestimation, as $67 \%$ of the data showed underestimation, while overestimation was shown in $33 \%$ of the data. Position of $g m p$ in the $\mathrm{z}$ axis was comparable across all sizes for both objects, mean GDisp z $_{\mathrm{z}}$ ranged from $-34.34 \mathrm{~mm}(\mathrm{SD}=65.58)$ to $-42.13 \mathrm{~mm}$ $(\mathrm{SD}=55.54)$ in all cube sizes, and from $-27.04 \mathrm{~mm}(\mathrm{SD}=61.96)$ to $-31.86 \mathrm{~mm}(\mathrm{SD}=62.98)$ in all sphere sizes. However, high variation in $G D i s p_{\mathrm{z}}$, as shown by the high SD values, for both objects was present. Across all sizes, participants had lower mean GDisp in grasping spheres $(M=-29.87 \mathrm{~mm}, \mathrm{SD}=60.50)$ than cubes $(M=$ $-38.39 \mathrm{~mm}, \mathrm{SD}=61.67)$.

As statistically significant results were found for both size and object types conditions, the null hypothesis that the conditions do not have an effect on GDisp in all three axes (GDisp $p_{\mathrm{x}}, G D i s p_{\mathrm{y}}$ and $\left.G D i s p_{\mathrm{z}}\right)$ is rejected, as GDisp is affected by changes in size and object type.

Even though gmp placement across all axes was comparable across sizes, grasp variations in terms of orientation and type was present in between participants as shown in Fig. 5, indicating that participants adapted their medium wrap grasp that they were instructed to use to different grasp types with changes in object size.

\section{Experiment 2: Object Position and Shape}

We used a $2 \times 3 \times 3 \times 3$ within-subjects design, with two primary conditions: object position and object type (see Table 1). All 15 new participants took part in both conditions (again cube and sphere). Every permutation of position was randomly presented to participants to exclude potential learning effects. In total, each participant completed 27 (positions) $\times 2$ (objects) $=54$ trials and 810 grasps (54 trials $\times 15$ participants). Each static grasp of every participant was recorded for 5 seconds ( 75 frames), leading to collecting 60750 raw data points ( 810 grasps $\times 75$ frames).

Hypothesis: We test the null hypotheses that changes in object position have no effect on a) Grasp Aperture and b) Grasp Displacement

\subsection{Procedure}

Again participants were instructed to accurately locate and match their medium wrap Grasp Aperture to the size and position of the virtual object in the shortest time possible. For experiment 2, we have adapted the methodology of Stockmeier et al. [36], thus 27 different positions in all axes (x,y and $\mathrm{z}$ ) are used (see Table 1), covering a working range of $400 \mathrm{~mm}$ from participants (see Fig. $3 \mathrm{~b}$ ). We chose the object sizes that had the lowest mean difference between $G A p$ and object size in Experiment $1(80 \mathrm{~mm}$ for cubes and $70 \mathrm{~mm}$ for spheres). Sizes were unchanged throughout the experiment. During the experiment, an object (cube or sphere) appeared to participants on the feedback monitor.

\subsection{Results And Analysis}

The object position that was used in Experiment 1 (Centre Middle) was changed in this experiment across the $\mathrm{x}, \mathrm{y}$ and $\mathrm{z}$ axes (see Table 1). We report on results of the $\mathrm{z}$ plane that was used in Experiment $1(1600 \mathrm{~mm})$, and changes in object position were compared to the Centre Middle position to asses the influence of position changes on GAp and GDisp. The influence of changes in the z plane on GAp and GDisp is analysed in the form of set comparisons $(1400 \mathrm{~mm}$ to $1600 \mathrm{~mm}, 1800 \mathrm{~mm}$ to $1400 \mathrm{~mm}$ and $1800 \mathrm{~mm}$ to $1600 \mathrm{~mm}$ ) and not individual positions. Full comparisons of all positions across all z planes are reported in Table 3.

\subsubsection{Results - Grasp Aperture (GAp)}

Statistically significant differences in Grasp Aperture (GAp) between different object types in different positions $\left(\chi^{2}(1)=636, p<\right.$ 0.01 ) were found. Statistically significant differences in $G A p$ between different cube positions $\left(\chi^{2}(8)=559, p<0.01\right)$ and sphere positions $\left(\chi^{2}(8)=2144, p<0.01\right)$ were also found. Significant adjusted post-hoc results are reported in Table 3 (see $\bullet$ for cubes, $\mathrm{O}$ for spheres).

For both objects, majority of effects in the multiple comparison were medium $(d>0.50)$ and large $(d>0.80)$.

Participants had the lowest mean difference between $G A p$ and object size in the Bottom Right position for cubes $(M=79.56 \mathrm{~mm}$, SD $=17.45)$ and spheres $(M=71.50 \mathrm{~mm}, \mathrm{SD}=16.72)$. Highest mean difference between $G A p$ and object size was present in the Top Left position for cubes $(M=87.91 \mathrm{~mm}, \mathrm{SD}=17.41)$ and spheres $(M=$ $85.36 \mathrm{~mm}, \mathrm{SD}=15.59)$.

\subsubsection{Findings - Grasp Aperture (GAp)}

For both objects, 6 positions have shown significant differences in comparisons with the Centre Middle position from Experiment 1, with effect sizes that ranged from medium effects $(d>0.50)$ to large effects $(d>0.80)$. This indicates that participants changed their $G A p$ in the majority of positions of objects. Two positions, Centre Right and Top Right, showed no significant differences in comparison to the Center Middle position, indicating statistical similarity of grasps between participants in those 3 positions, that potentially shows a working range which is preferable by participants.

Fig. 8 shows higher mean differences between $G A p$ and object size in positions that were to the left hand side of participants (Top Left, Centre Left and Bottom Left) for cubes $(M=84.44 \mathrm{~mm}$, SD $=14.89)$, and spheres $(M=83.31 \mathrm{~mm}, \mathrm{SD}=13.84)$. Lower mean differences between $G A p$ and object size were found in positions 
Table 3: Significant Post-hoc Pairwise Comparisons - Experiment 2

\begin{tabular}{|c|c|c|c|c|c|c|c|c|c|}
\hline \multicolumn{10}{|c|}{$\begin{array}{c}\text { Z Plane 1 - 1400mm } \\
\end{array}$} \\
\hline Positions & Top Left & Top Middle & Top Right & Centre Left & \begin{tabular}{|l|} 
Centre Middle \\
\end{tabular} & Centre Right & Bottom Left & Bottom Middle & \begin{tabular}{|l|} 
Bottom Right \\
\end{tabular} \\
\hline Top Left & & $\circ \Delta \Delta \diamond \diamond \square \square$ & $\bullet \circ \Delta \Delta \bullet \diamond \| \square$ & $\circ \Delta \Delta \diamond \diamond \square$ & $\bullet \circ \Delta \Delta \diamond \diamond \| \square$ & $\bullet \circ \Delta \Delta \bullet \diamond \mid \square$ & $\bullet \quad \Delta \Delta \bullet \diamond \square \square$ & $\circ \Delta \Delta \bullet \diamond \square$ & $\bullet \circ \Delta \Delta \bullet \diamond \square \square$ \\
\hline Top Middle & & & $\bullet \circ \Delta \Delta \diamond \| \square$ & $\bullet \circ \quad \Delta \bullet \diamond \quad \square$ & $\bullet \quad \Delta \bullet \diamond \square$ & $\bullet \circ \Delta \Delta \bullet \diamond \mathbf{\square}$ & $\bullet \circ \quad \Delta \bullet \diamond \| \square$ & $\bullet \circ \Delta \Delta \diamond \diamond \square \square$ & $\bullet \circ \Delta \Delta \bullet \diamond \mathbf{\square}$ \\
\hline Top Ris & ----7 & --- & & $\bullet \circ \Delta \Delta \bullet \diamond$ & $\circ \Delta \Delta \bullet \diamond$ & $\circ \Delta \Delta \diamond \diamond \quad \square$ & $\bullet \circ \Delta \Delta \bullet \diamond \| \square$ & $\bullet \circ \Delta \Delta \diamond \diamond \quad \square$ & $\bullet \circ \Delta \Delta \bullet \diamond \square$ \\
\hline Centre Left & ---- & - - - - - - - - & & & $\bullet \circ \quad \Delta \diamond \diamond$ & $\bullet \circ \Delta \Delta \diamond$ & $\circ \diamond \diamond \square$ & $\circ \Delta \Delta \diamond \diamond$ & \\
\hline Centre Middle & ----1 & ------ & -- & & & $0 \Delta \Delta \diamond \diamond$ & $\Delta \diamond \diamond \quad \square$ & $\bullet \circ \diamond \diamond$ & $\bullet \circ \Delta \Delta \diamond \Delta$ \\
\hline Centre & -----1 & ------- & ------ & -- & & & $\bullet \circ \Delta \Delta \triangleleft \Delta$ & - $0 \Delta \Delta \Delta \Delta$ & $\bullet \circ \quad \diamond \Delta \square$ \\
\hline Bottom Left & -----1 & -----1 & ---- & & -- & & & $\bullet \quad \Delta \Delta \bullet$ & $\circ \Delta \Delta$ \\
\hline & ---7 - & - - & -- & - & & & & & $\bullet \circ \Delta \Delta$ \\
\hline & & & & & & & & & \\
\hline \multicolumn{10}{|c|}{ Z Plane 2 - 1600mm } \\
\hline Positions & Top Left & Top Middle & Top Right & Centre Left & Centre Middle & \begin{tabular}{|c|} 
Centre Right \\
\end{tabular} & Bottom Left & Bottom Middle & Bottom Right \\
\hline Top Left & & $\bullet \circ \Delta \Delta \diamond \diamond$ & $\bullet \circ \Delta \Delta \bullet \diamond \square \square$ & $\bullet \circ \Delta \bullet \diamond \square$ & $\bullet \circ \Delta \Delta \bullet \diamond \square$ & $\bullet \circ \Delta \Delta \bullet \diamond \square \square$ & $\bullet \quad \Delta \Delta \bullet \diamond \square \square$ & $\bullet \circ \Delta \Delta \diamond \diamond \square$ & $\bullet \circ \Delta \Delta \diamond \diamond \square \square$ \\
\hline Top M & & & $\bullet \circ \Delta \Delta \bullet \quad \square$ & $\bullet \circ \quad \Delta \bullet \diamond \| \square$ & $\bullet \circ \quad \Delta \diamond \diamond \quad \square$ & $\circ \Delta \Delta \diamond \diamond \bar{\square}$ & $\bullet \circ \quad \Delta \bullet \diamond \| \square$ & $\bullet \quad \Delta \bullet \diamond \square$ & $\bullet \circ \Delta \Delta \diamond \diamond \|$ \\
\hline Top Right & & & & 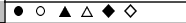 & $\Delta \Delta \diamond \diamond$ & $\circ \Delta \Delta \diamond \diamond \| \square$ & $\bullet \circ \Delta \Delta \bullet \diamond \| \square$ & $\bullet \circ \Delta \Delta \diamond \diamond \square \square$ & $\bullet \circ \Delta \Delta \diamond \diamond \square$ \\
\hline Centre Left & ---- & ---- & & & $\bullet \circ \quad \Delta \quad \diamond \| \square$ & $\bullet \circ \Delta \Delta \bullet \diamond \square \square$ & $\circ \quad \diamond \diamond \square$ & $\circ \Delta \Delta \diamond \diamond \square$ & $\circ \Delta \Delta \diamond \diamond$ \\
\hline Centre & $=---1$ & -----1 & --1 & & & 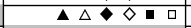 & $\bullet \circ \quad \Delta \bullet \diamond \| \square$ & $\bullet \circ \quad \Delta \bullet \diamond \square$ & $\bullet \circ \Delta \Delta \diamond \diamond \| \square$ \\
\hline Centre Right & ---- & & & & & & $\bullet \circ \Delta \Delta \diamond \diamond \quad \square$ & $\bullet \circ \Delta \Delta \bullet \diamond \quad \square$ & $\bullet \circ \Delta \bullet \diamond \square$ \\
\hline Bottor & ----1 & ---- & - & & 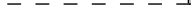 & & & $0 \boldsymbol{\Delta} \Delta \Delta \boldsymbol{\|}$ & $\circ \mathbf{\Delta} \Delta \diamond \diamond$ \\
\hline & ---1 & -- & & 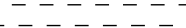 & --- & & & & $\Delta \bullet \quad \square$ \\
\hline & & & & & & & & & \\
\hline \multicolumn{10}{|c|}{ Z Plane 3 - 1800mm } \\
\hline Positions & Top Left & Top Middle & Top Right & $\begin{array}{l}\text { Centre Left } \\
\end{array}$ & Centre Middle & Centre Right & Bottom Left & Bottom Middle & Bottom Right \\
\hline Top Left & & - $\circ \Delta \Delta \diamond \Delta \square$ & $\bullet \circ \Delta \Delta \quad \diamond \quad \square$ & $\bullet \circ \Delta \Delta \diamond \diamond \square \square$ & $\bullet \circ \Delta \Delta \bullet \diamond \| \square$ & $\bullet \circ \Delta \Delta \bullet \diamond \square \square$ & $\bullet \circ \quad \Delta \bullet \diamond \| \square$ & $\bullet \circ \Delta \Delta \bullet \diamond \square \square$ & $\bullet \circ \Delta \Delta \diamond \diamond \| \square$ \\
\hline Top Middle & -- & & $\bullet \circ \Delta \Delta$ & $\bullet \circ \Delta \Delta \bullet \diamond \square \square$ & $\bullet \circ \Delta \bullet \diamond \square \square$ & $\bullet \circ \Delta \Delta \bullet \diamond \quad \square$ & $\Delta \Delta \bullet \diamond \square$ & $\bullet \circ \Delta \bullet \diamond \mathbf{\Delta}$ & $\bullet \circ \Delta \Delta \bullet \diamond \square \square$ \\
\hline Top Right & -- & $--\ldots--\ldots$ & & $\bullet \circ \Delta \Delta \diamond \diamond \square$ & $\bullet \circ \Delta \Delta \bullet \diamond$ & $\bullet \quad \Delta \Delta \bullet \diamond \| \square$ & $\bullet \circ \Delta \Delta \bullet \diamond$ & $\bullet \circ \Delta \Delta \bullet \diamond \mathbf{I}$ & $\bullet \circ \Delta \Delta \diamond \diamond \square$ \\
\hline Centre Left & -- & & & & $\bullet \circ \Delta \Delta$ & $\bullet \circ \Delta \Delta \quad \diamond \quad \square$ & $\bullet \circ \quad \diamond \diamond \| \square$ & $\bullet \circ \Delta \Delta \diamond \diamond \| \square$ & $\circ \Delta \Delta \diamond \diamond$ \\
\hline Centre Middle & -- & $-1-1--$ & ---- & & & $O \Delta \Delta$ & $\bullet \circ \Delta \Delta \bullet \diamond \square$ & $\diamond \diamond \square$ & $\bullet \circ \Delta \Delta \diamond \diamond \square \square$ \\
\hline Centr & & - & - & & & & $\bullet \circ \Delta \Delta \diamond \diamond \| \square$ & $\bullet \circ \Delta \Delta \bullet \diamond \square$ & $\Delta \Delta \diamond \diamond$ \\
\hline Bottom Left & & & & & & & & $\circ \Delta \Delta \bullet$ & $\bullet \circ \Delta \triangle$ \\
\hline & & & & & 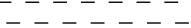 & -- & & & - $\quad \Delta \Delta$ \\
\hline & & & & & & & & & \\
\hline
\end{tabular}

Symbols represented in constant order ( $\bullet \Delta \Delta \diamond \diamond \square \square)$ show significance in a post-hoc Dunn Test with Bonferroni correction using an $\alpha$ level of 0.01 for the following: $\bullet$ GAp - Cube, $\bigcirc$ GAp - Sphere, $\Delta$ GDisp $p_{\mathrm{x}}-$ Cube, $\triangle$ GDisp $p_{\mathrm{x}}-\mathrm{Sphere}_{\boldsymbol{B}} \diamond$ GDisp $p_{\mathrm{y}}-$ Cube, $\diamond G D i s p_{\mathrm{y}}-$ Sphere, GDisp $_{\mathrm{z}}$ - Cube, $\square$ GDisp $\mathrm{z}$ - Sphere. No symbols indicate statistical similarity. For positions, see Table 1

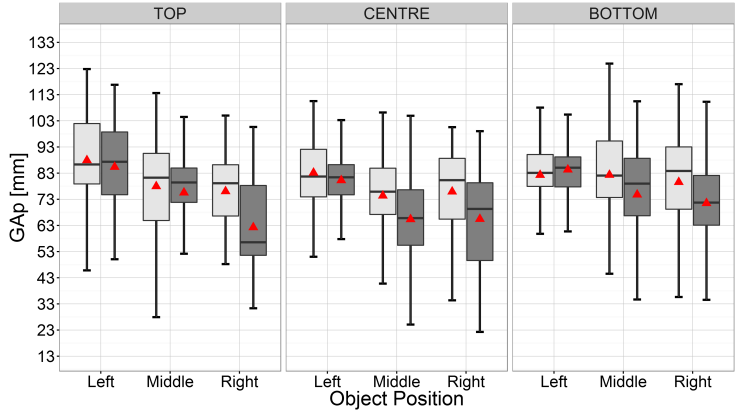

Figure 8: GAp for different object positions in the $1600 \mathrm{~mm} \mathrm{z}$ plane. Light grey boxplots show cubes, and dark grey boxplots show spheres. Red triangles on boxplots indicate the mean GAp across all participants for each size. Whiskers represent the highest and lowest values within 1.5 times the interquartile range from the lower and upper quartiles. For positions, see Table 1

to the right hand side of participants (Top Right, Centre Right and Bottom Right) for cubes $(M=77.17 \mathrm{~mm}, \mathrm{SD}=15.90)$, and spheres $(M=66.40 \mathrm{~mm}, \mathrm{SD}=17.03)$. Central positions (Top Middle, Centre Middle and Bottom Middle) presented the lowest mean differences between $G A p$ and object size for cubes $M=78.21 \mathrm{~mm}, \mathrm{SD}=17.40$ ), and spheres $(M=71.88 \mathrm{~mm}, \mathrm{SD}=17.52)$.

Overestimation of object size was present in positions on the left hand side of participants. Mean overestimation of $4.44 \mathrm{~mm}$ (SD $=14.89)$ for cubes and $13.31 \mathrm{~mm}(\mathrm{SD}=13.84)$ for spheres was present. Underestimation was consistent in positions on the right hand side of participants. Mean underestimation of $-2.83 \mathrm{~mm}$ (SD $=15.90)$ for cubes and $-3.60 \mathrm{~mm}(\mathrm{SD}=17.03)$ for spheres was present.

Statistically significant differences in $G A p$ between different $\mathrm{z}$ planes for cubes were found $\left(\chi^{2}(2)=458, p<0.01\right)$. This shows that participants altered their $G A p$ as position of objects changed in the $\mathrm{z}$ axis when grasping cubes. No statistically significant differences were found in $G A p$ between different $\mathrm{z}$ planes for spheres $\left(\chi^{2}(2)=3.63, p>0.01\right)$, indicating that participants had comparable $G A p$ across all z planes when grasping spheres.

Mean completion time ranged from $4.46(\mathrm{SD}=1.96)$ to $8.46(\mathrm{SD}$ $=8.46$ ) seconds for both objects. Even though statistically significant differences in completion time between different cube positions $\left(\chi^{2}(8)=380, p<0.01\right)$ and sphere positions $\left(\chi^{2}(8)=739\right.$, $p<0.01)$ were found, all effect sizes were small $(d<0.30)$ or negligible $(d<0.20)$, and no trends between completion time and object size were found.

As statistically significant results were found for both position and object types conditions, the null hypothesis that the conditions do not have an effect on $G A p$ is rejected. GAp is affected by changes in position and object type, within the bounds of the range found $(66.40 \mathrm{~mm}$ to $84.44 \mathrm{~mm})$.

\subsubsection{Results - Grasp Displacement (GDisp)}

Statistically significant differences in Grasp Displacement in the $\mathrm{x}$ axis $\left(G D i s p_{\mathrm{x}}\right)$ between different object types in different positions $\left(\chi^{2}(1)=456, p<0.01\right)$ were found. Statistically significant differences in GDisp $p_{\mathrm{x}}$ between different cube positions $\left(\chi^{2}(8)=1954\right.$, $p<0.01)$ and sphere positions $\left(\chi^{2}(8)=3251, p<0.01\right)$ were also found. Significant adjusted post-hoc results are reported in Table 3 (see $\boldsymbol{\Delta}$ for cubes, $\Delta$ for spheres).

For both objects, the majority of effects in the multiple comparison were large $(d>0.80)$.

Participants had the lowest mean GDisp $p_{\mathrm{x}}$ in the Top Right position for cubes $(M=5.94 \mathrm{~mm}, \mathrm{SD}=30.31)$, and spheres $(M=-2.96 \mathrm{~mm}$, $\mathrm{SD}=24.53)$. Highest mean GDisp $p_{\mathrm{x}}$ was present in the Top Left position for cubes $(M=52.28 \mathrm{~mm}, \mathrm{SD}=26.93)$, and spheres $(M=$ $47.93 \mathrm{~mm}, \mathrm{SD}=33.40)$.

Statistically significant differences in Grasp Displacement in the $y$ axis $\left(G D i s p_{\mathrm{y}}\right)$ between different object types in different positions $\left(\chi^{2}(1)=12, p<0.01\right)$ were found. Statistically significant differences in GDisp $p_{\mathrm{y}}$ between different cube positions $\left(\chi^{2}(8)=3873\right.$, $p<0.01)$ and sphere positions $\left(\chi^{2}(8)=4174, p<0.01\right)$ were also found. Significant adjusted post-hoc results are reported in Table 3 (see $\diamond$ for cubes, $\diamond$ for spheres). 
For both objects, the majority of effects in the multiple comparison were large $(d>0.80)$.

Participants had the lowest mean GDisp $p_{\mathrm{y}}$ in the Bottom Right position for cubes $(M=2.35 \mathrm{~mm}, \mathrm{SD}=20.22)$, and in the Centre Right position for spheres $(M=-2.96 \mathrm{~mm}, \mathrm{SD}=15.14)$. Highest mean GDisp y $_{\mathrm{y}}$ was present in the Top Left position for cubes $(M=$ -34.62mm, $\mathrm{SD}=20.65)$, and spheres $(M=-35.15 \mathrm{~mm}, \mathrm{SD}=23.21)$. Statistically significant differences in Grasp Displacement in the $\mathrm{z}$ axis $\left(G D i s p_{\mathrm{z}}\right)$ between different object types in different positions $\left(\chi^{2}(1)=16, p<0.01\right)$ were found. Statistically significant differences in $G D i s p_{\mathrm{z}}$ between different cube positions $\left(\chi^{2}(8)=1218\right.$, $p<0.01)$ and sphere positions $\left(\chi^{2}(8)=1455, p<0.01\right)$ were also found. Significant adjusted post-hoc results are reported in Table 3 (see $\square$ for cubes, $\square$ for spheres).

For both objects, the majority of effects in the multiple comparison were medium $(d>0.50)$ and small $(d>0.20)$.

Participants had the lowest mean GDisp $p_{\mathrm{z}}$ in the Bottom Right position for cubes $(M=7.28 \mathrm{~mm}, \mathrm{SD}=38.87)$, and spheres $(M=$ $4.77 \mathrm{~mm}, \mathrm{SD}=30.20)$. Highest mean GDisp $p_{\mathrm{z}}$ was present in the Top Left position for cubes $(M=129.32 \mathrm{~mm}, \mathrm{SD}=105.30)$, and spheres $(M=106.78 \mathrm{~mm}, \mathrm{SD}=107.01)$.

\subsubsection{Findings - Grasp Displacement (GDisp)}

In Top Middle, Centre Left, Bottom Left, Bottom Middle and Bottom Right positions (cube), participants had statistically similar $G D i s p_{\mathrm{x}}$ to the Center Middle position. This shows that that change of position did not have an influence on GDisp $p_{\mathrm{x}}$ in the majority of positions across all participants. Remaining positions resulted in significant comparisons with the Center Middle position, with effect sizes that ranged from small $(d<0.30)$ to large $(d>0.80)$. All position comparisons with the Centre Middle position for the sphere have shown significant differences in GDisp $p_{\mathrm{x}}$, with effect sizes that ranged from medium effects $(d>0.30)$ to large effects $(d>0.80)$.

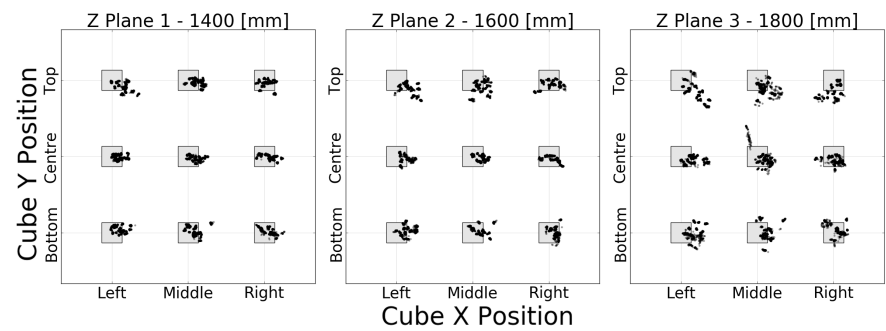

(a)

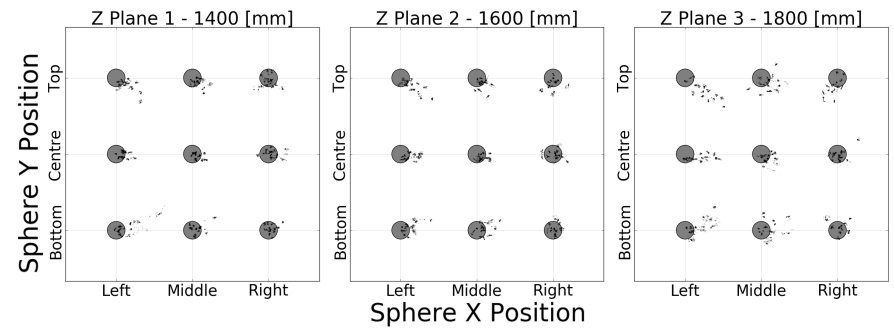

(b)

Figure 9: gmp placement (black clusters) in the $\mathrm{x}$ and $\mathrm{y}$ axes of all participants in 27 positions in $3 \mathrm{z}$ planes $(1400 \mathrm{~mm}, 1600 \mathrm{~mm}$ and $1800 \mathrm{~mm}$ ) for Experiment 2: (a) cube, and (b) sphere

Mean GDisp $p_{\mathrm{x}}$ ranged from $5.94 \mathrm{~mm}(\mathrm{SD}=30.31)$ to $52.28 \mathrm{~mm}(\mathrm{SD}$ $=26.93)$ for cubes, and from $-2.96 \mathrm{~mm}(\mathrm{SD}=24.53)$ to $47.93 \mathrm{~mm}$ $(\mathrm{SD}=33.40)$ for spheres as shown by clusters in Fig. 9a and Fig. 9b. This range for both objects shows that even though participants showed statistical similarities in $g m p$ placement for cubes, GDisp $p_{\mathrm{x}}$ shows variability across participants.

Participants had statistically similar GDis $p_{\mathrm{y}}$ in the Centre Left position to the Center Middle position. Remaining positions resulted in significant comparisons with the Centre Middle position, with effect sizes that ranged from small $(d<0.30)$ to large $(d>0.80)$. All position comparisons with the Centre Middle position for the sphere have shown significant differences in GDisp $p_{\mathrm{y}}$, with effect sizes that ranged from medium effects $(d>0.30)$ to large effects $(d>0.80)$

Negative GDisp $p_{\mathrm{y}}$ was found across all participants in all positions for both objects, with the exception of positions Bottom Left, Bottom Middle and Bottom Right. Participants placing their gmp to a point that is lower than the $o m p$ was more common.

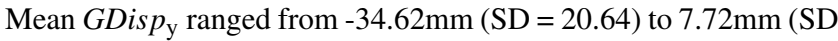
$=17.47)$ for cubes, and from $-35.15 \mathrm{~mm}(\mathrm{SD}=23.21)$ to $10.29 \mathrm{~mm}$ $(\mathrm{SD}=20.18)$ for spheres as shown by clusters in Fig. 9a and Fig. 9b. Wide ranges across participants and objects show variability in $g m p$ placement in the y axis.

Statistically similar GDisp $p_{\mathrm{z}}$ to the Centre Middle position was found in the Top Middle position. Remaining positions resulted in significant comparisons with the Centre Middle position, with effect sizes ranging from small $(d<0.30)$ to large $(d>0.80)$. All position comparisons with the Centre Middle position for the sphere have shown significant differences in $G D i s p_{\mathrm{z}}$, with effect sizes ranging from medium effects $(d>0.30)$ to large effects $(d>0.80)$.

Changes in position have noticeably increased the mean GDisp $p_{\mathrm{z}}$ in comparison to Experiment 1 as it ranged from $-129.32 \mathrm{~mm}$ (SD = $105.30)$ to $-7.28 \mathrm{~mm}(\mathrm{SD}=38.87)$ for cubes, and from $-106.78 \mathrm{~mm}$ $(\mathrm{SD}=107.01)$ to $4.77 \mathrm{~mm}(\mathrm{SD}=30.20)$ for spheres. Depth estimation in freehand grasping is problematic due to lack of feedback cues, high SD in depth estimation in different positions highlight this problem, that can potentially be reduced through the use of multiple views for visual feedback.

As shown in Fig. 10a and Fig. 10b, the majority of participants have underestimated the position of objects in the $\mathrm{z}$ axis. Meaning that participants placed their $g m p$ in front of the omp. This underestimation has also shown to decrease as objects were further away from participants ( $\mathrm{z}$ plane $1400 \mathrm{~mm}$ ). It can be argued that participants were more accurate in depth estimation as objects were further away from them. However, margin of error in the furthest $\mathrm{z}$ plane was limited to the mean arm length of participants and the amount of movement permitted within the test.

High displacement values in all axes $\left(G D i s p_{\mathrm{x}}, G D i s p_{\mathrm{y}}\right.$ and $G D i s p_{\mathrm{Z}}$ ) in positions on the left hand side of participants were found. Conversely, low displacement values in positions on the right hand side of participants were present.

Between different $\mathrm{z}$ planes, statistically significant differences in GDis $p_{\mathrm{x}}$ between different $\mathrm{z}$ planes for cubes $\left(\chi^{2}(2)=114, p<\right.$ $0.01)$ and spheres $\left(\chi^{2}(2)=162, p<0.01\right)$ were found. Differences in GDis $p_{\mathrm{y}}$ were also statistically significant for cubes $\left(\chi^{2}(2)\right.$ $=1286, p<0.01)$ and sphere $\left(\chi^{2}(2)=472, p<0.01\right)$. Statistically significant differences in GDisp $p_{\mathrm{Z}}$ were also found for cubes $\left(\chi^{2}(2)\right.$ $=3376, p<0.01)$, and spheres $\left(\chi^{2}(2)=3104, p<0.01\right)$. This shows that participants altered their $g m p$ placement as position of objects changed in the $\mathrm{z}$ axis.

As statistically significant results were found for both position and object types conditions, the null hypothesis that the conditions do not have an effect on GDisp in all three axes (GDisp $p_{\mathrm{x}}, G D i s p_{\mathrm{y}}$ and $G D i s p_{\mathrm{Z}}$ ) is rejected. Due to large variations in $g m p$ placement for both objects in different positions, GDisp is affected by changes in size and object type.

Changing object position has opted participants to adapt their grasp posture in terms of dexterity and type as shown in Fig. 11. Change 

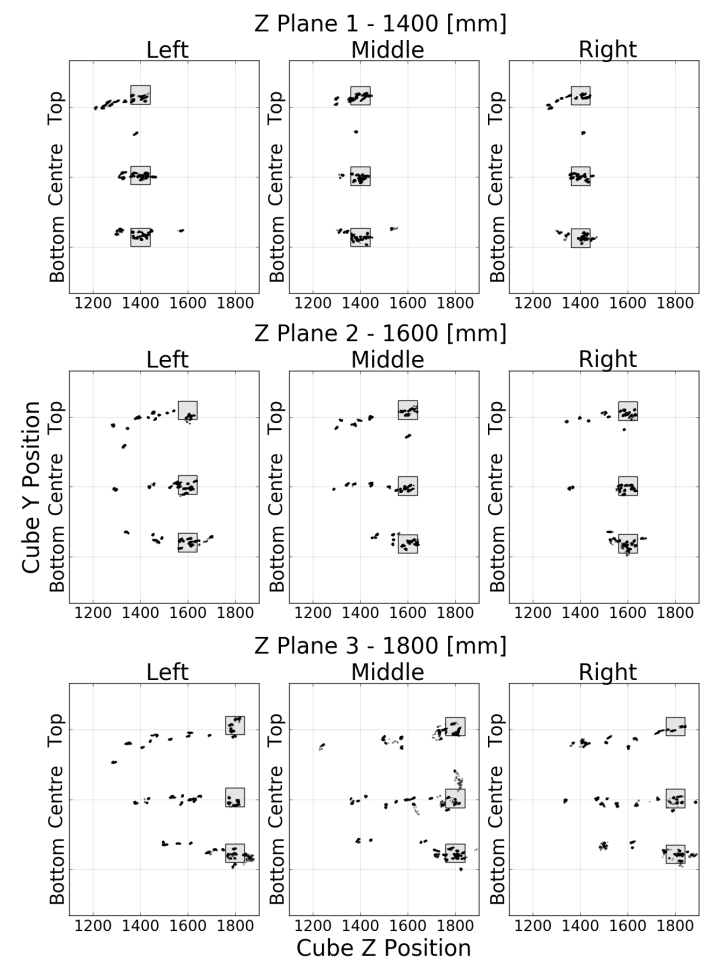

(a)
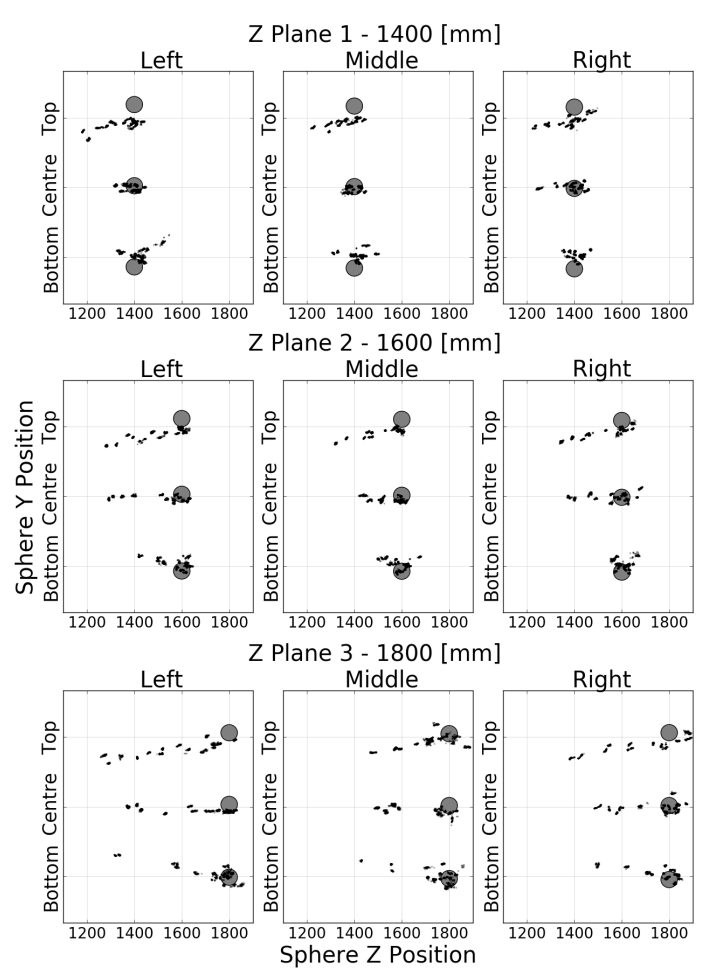

(b)

Figure 10: gmp placement (black clusters) in the $\mathrm{z}$ axis of all participants in 27 positions in $3 \mathrm{z}$ planes $(1400 \mathrm{~mm}, 1600 \mathrm{~mm}$ and $1800 \mathrm{~mm})$ for Experiment 2: (a) cube, and (b) sphere

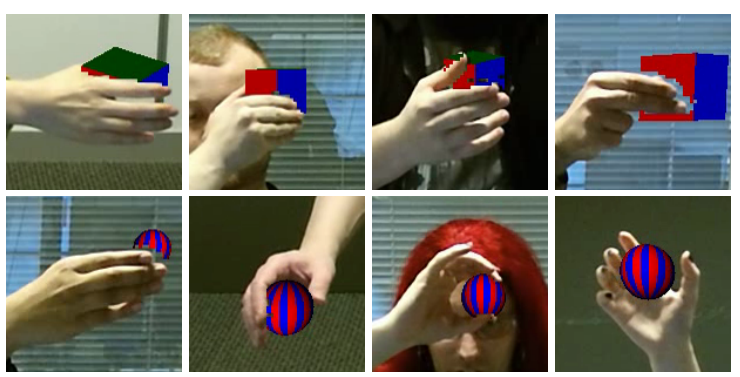

Figure 11: Examples of grasp variation in Experiment 2 (cubes and spheres) for different positions in 3D space

of grasp type was present in both experiments, indicating that object size, position and shape influence grasp choice in freehand grasping. This problem shows that classifications of grasps for virtual objects requires further exploration and extension beyond the taxonomy presented in $[14,15]$.

\section{CONCLUSIONS AND FUtURE WORK}

Findings from this work present the first study into the range of user accuracy for freehand grasping in exocentric MR. We present a comprehensive study detailing inherent problems users face when attempting a common grasp (medium wrap) on a virtual object and we report using two new measures of grasp ability namely, Grasp Aperture $(G A p)$ and Grasp Displacement $\left(G D i s p_{\mathrm{xyz}}\right)$. Throughout the analysis we have presented a rigorous experimental methodology combined with a comprehensive pairwise statistical evaluation, which illustrates the user trends when changes are made to the virtual object size, position and shape. We illustrate the similarity across users when estimating virtual object size and report on the commonality in Grasp Aperture $(G A p)$. Notably that $G A p$ varies less than expected from physical studies and that it does not increase directly proportional to increases in object size. Furthermore commonalities were seen in GAp across users, showing common aperture sizes irrespective of object size. However we have shown that even when instructed to complete a controlled medium wrap grasp, user grasp varies considerably. These findings are important when understanding how users grasp virtual objects and noteworthy for future work developing freehand grasping systems. Our results also illustrate that the freehand Grasp Displacement (GDisp) is greater in the $\mathrm{z}$ axis when compared to the $\mathrm{x}$ and $\mathrm{y}$ axes positions and users commonly under judge the object's $\mathrm{z}$ position. These findings can be attributed to the conditions under test, notably the feedback method employed and the grasp method chosen. Occlusion between the user's hand and parts of the virtual object was an indicator of their grasp accuracy thus provides a visual stimulus for users assessing their own grasp accuracy. Again this underestimation should be considered within interaction design since under judging the $\mathrm{z}$ potion of an object while being correct in $\mathrm{x}$ and $\mathrm{y}$ potion could allow interactions to remain visually credible. Finally, we have confirmed that freehand grasping performance is superior when grasping on the right and centre regions in front of participants. This was attributed to the dominant hand within our subjects, however it does indicate that defining an interaction range to the centre and the side of the dominant hand can lead to improvements grasping placement. Future work will consider the changes to grasp accuracy when using different virtual object rendering and feedback methods. Furthermore, implementations of interaction utilising the results from this study, notably the GAp similarity and GDisp variances will aim to provide a more dynamic and potentially more intuitive interface between virtual objects and the human hand. 


\section{REFERENCES}

[1] S. Suzuki, H. Suzuki, and M. Sato, "Grasping a virtual object with a bare hand," ACM SIGGRAPH 2014 Posters, pp. 1-1, 2014.

[2] D. Holz, S. Ullrich, M. Wolter, and T. Kuhlen, "Multi-Contact Grasp Interaction for Virtual Environments," Journal of Virtual Reality and Broadcasting, vol. 5, no. 7, pp. 101-112, 2008.

[3] K. Ponto, R. Kimmel, J. Kohlmann, A. Bartholomew, and R. G. Radwir, "Virtual exertions: A user interface combining visual information, kinesthetics and biofeedback for virtual object manipulation," Proceedings - IEEE Symposium on $3 D$ User Interfaces, pp. 85-88, 2012.

[4] J. Rekimoto, "Smartskin: an infrastructure for freehand manipulation on interactive surfaces," Proceedings - SIGCHI conference on Human factors in computing systems, vol. 02, pp. 113-120, 2002.

[5] M. Wu and R. Balakrishnan, "Multi-finger and whole hand gestural interaction techniques for multi-user tabletop displays," Proceedings - ACM symposium on User interface software and technology, vol. 5, no. 2, pp. pages 193-202, 2003.

[6] H. Benko, R. Jota, and A. Wilson, "MirageTable: Freehand Interaction on a Projected Augmented Reality Tabletop," Proceedings - ACM annual conference on Human Factors in Computing Systems, pp. 199-208, 2012.

[7] T. Feix, R. Pawlik, H. B. Schmiedmayer, J. Romero, and D. Kragi, “A comprehensive grasp taxonomy," Robotics, Science and Systems Conference: Workshop on Understanding the Human Hand for Advancing Robotic Manipulation, pp. 2-3, 2009.

[8] C. L. MacKenzie and T. Iberall, The grasping hand. Elsevier, 1994, vol. 104

[9] M. R. Cutkosky and R. D. Howe, "Human Grasp Choice and Robotic Grasp Analysis," Dextrous Robot Hands, vol. 1, pp. 5-31, 1990.

[10] N. Kamakura, M. Matsuo, H. Ishii, F. Mitsuboshi, and Y. Miura, "Patterns of static prehension in normal hands," American Journal of Occupational Therapy, vol. 34, no. 7, pp. 437-445, 1980.

[11] D. M. Lyons, "A simple set of grasps for a dextrous hand," Proceedings - IEEE International Conference on Robotics and Automation, vol. 2, pp. 588-593, 1985.

[12] T. Iberall, "The nature of human prehension: Three dextrous hands in one," Proceedings - IEEE International Conference on Robotics and Automation, vol. 4, pp. 396-401, 1987.

[13] M. R. Cutkosky, "On grasp choice, grasp models, and the design of hands for manufacturing tasks," IEEE Transactions on Robotics and Automation, vol. 5, no. 3, pp. 269-279, 1989.

[14] I. M. Bullock, T. Feix, and A. M. Dollar, "Finding small, versatile sets of human grasps to span common objects," Proceedings - IEEE International Conference on Robotics and Automation, pp. 1068-1075, 2013.

[15] T. Feix, I. M. Bullock, and A. M. Dollar, "Analysis of Human Grasping Behavior: Correlating Tasks, Objects and Grasps," IEEE Transactions on Haptics, vol. 7, no. 3, pp. 311-323, 2014.

[16] M. Lee, G. A and Billinghurst and G. J. Kim, "Occlusion based interaction methods for tangible augmented reality environments," Virtual Reality Continuum And Its Applications, vol. 1, no. 212, p. 419, 2004.

[17] V. Buchmann, S. Violich, M. Billinghurst, and A. Cockburn, "FingARtips: gesture based direct manipulation in Augmented Reality," Proceedings - International conference on
Computer graphics and interactive techniques in Australasia and South East Asia, pp. 212-221, 2004.

[18] H. Bai, L. Gao, J. El-Sana, and M. Billinghurst, "Markerless $3 \mathrm{D}$ gesture-based interaction for handheld Augmented Reality interfaces," IEEE ISMAR, pp. 1-6, 2013.

[19] D. Datcu and S. Lukosch, "Free-hands interaction in augmented reality," Proceedings - Symposium on Spatial User Interaction, pp. 33-40, 2013.

[20] M. A. Cidota, R. M. S. Clifford, P. Dezentje, S. G. Lukosch, and P. J. M. Bank, "Affording Visual Feedback for Natural Hand Interaction in AR to Assess Upper Extremity Motor Dysfunction," IEEE ISMAR, pp. 1-4, 2015.

[21] J. E. Swan, J. L. Gabbard, and M. Hall, "Survey of user-based experimentation in augmented reality," Proceedings - International Conference on Virtual Reality, pp. 1-9, 2005.

[22] A. Dünser, R. Grasset, and M. Billinghurst, A survey of evaluation techniques used in augmented reality studies. Human Interface Technology Laboratory New Zealand, 2008.

[23] J. E. Swan, G. Singh, and S. R. Ellis, "Matching and Reaching Depth Judgments with Real and Augmented Reality Targets," IEEE TVCG, vol. 21, no. 11, pp. 1289-1298, 2015.

[24] G. Hough, I. Williams, and C. Athwal, "Fidelity and plausibility of bimanual interaction in mixed reality," IEEE TVCG, vol. 21, no. 12, pp. 1377-1389, 2015.

[25] R. P. McMahan, D. A. Bowman, D. J. Zielinski, and R. B. Brady, "Evaluating display fidelity and interaction fidelity in a virtual reality game," IEEE TVCG, vol. 18, no. 4, pp. 626633, 2012.

[26] D. Jack, R. Boian, A. S. Merians, M. Tremaine, G. C. Burdea, S. V. Adamovich, M. Recce, and H. Poizner, "Virtual reality-enhanced stroke rehabilitation," IEEE Transactions on Neural Systems and Rehabilitation Engineering, vol. 9, no. 3, pp. 308-318, 2001.

[27] A. Edsinger and C. C. Kemp, "Human-robot interaction for cooperative manipulation: Handing objects to one another,' IEEE International Symposium on Robot and Human interactive Communication, pp. 1167-1172, 2007.

[28] R. Oldfield, "The assessment and analysis of handedness: the Edinburgh inventory," Neuropsychologia, vol. 9, no. 1, pp. 97-113, 1971

[29] W. H. Kruskal and W. A. Wallis, "Use of ranks in onecriterion variance analysis," Journal of the American statistical Association, vol. 47, no. 260, pp. 583-621, 1952.

[30] A. Field, Discovering statistics using $R$. Sage publications, 2012.

[31] O. J. Dunn, "Multiple Comparisons Among Means," Journal of the American Statistical Association, vol. 56, no. 293, pp. 52-64, 1961.

[32] R. L. Iman and C. W. J., "Multiple comparisons procedures based on the rank transformation," Proceedings - Joint Statistical Meetings, Houston Texas, August, 1983.

[33] T. J. Terpstra, "The asymptotic normality and consistency of Kendall's test against trend, when ties are present in one ranking," Indagationes Mathematicae, vol. 14, no. 3, pp. 327-333, 1952.

[34] A. R. Jonckheere, "A distribution-free k-sample test against ordered alternatives," Biometrika, vol. 41, no. 1/2, pp. 133145,1954

[35] J. Cohen, “A power primer." Psychological Bulletin, vol. 112, no. 1, pp. 155-159, 1992.

[36] K. Stockmeier, H. Horton, and V. H. Franz, "How do we grasp (virtual) objects in three-dimensional space?" Journal of Vision, vol. 3, no. 9, pp. 383-383, 2003. 